\title{
Average-case complexity of shortest-paths problems in the vertex-potential model*
}

\author{
Colin Cooper $^{\dagger} \quad$ Alan Frieze $^{\ddagger} \quad$ Kurt Mehlhorn $^{\S} \quad$ Volker Priebe $^{\S}$
}

\begin{abstract}
We study the average-case complexity of shortest-paths problems in the vertex-potential model. The vertex-potential model is a family of probability distributions on complete directed graphs with arbitrary real edge lengths but without negative cycles. We show that on a graph with $n$ vertices and with respect to this model, the single-source shortest-paths problem can be solved in $O\left(n^{2}\right)$ expected time, and the all-pairs shortest-paths problem can be solved in $O\left(n^{2} \log n\right)$ expected time.
\end{abstract}

\section{Introduction}

A large variety of combinatorial-optimization problems can be modeled as shortest-paths problems. Given a (directed) graph in which edges are assigned real edge lengths, these problems ask for distances between pairs of vertices. The distance of vertex $j$ from vertex $i$ is defined as the infimum of the lengths of all directed paths from $i$ to $j$, where the length of a path is the sum of the lengths of its edges. (We need to take the infimum, since in the presence of a negative cycle, that is, a (directed) cycle of negative length, a finite minimum does not always exist.) We concentrate on two types of shortest-paths problems. In the single-source shortest-paths problem, we are interested in the distances of all vertices from a given source vertex; in the

*A preliminary version of this paper was presented at the International Workshop RANDOM'97, Bologna, Italy [6].

${ }^{\dagger}$ School of Mathematical Sciences, University of North London, London N7 8DB, U.K.

${ }^{\ddagger}$ Department of Mathematical Sciences, Carnegie Mellon University, Pittsburgh PA 15213, U.S.A. Research supported by NSF grant CCR-9225008.

${ }^{\S}$ Max-Planck-Institut für Informatik, Im Stadtwald, D-66123 Saarbrücken, Germany. Research partially supported by ESPRIT LTR Project no. 20244 - ALCOM-IT. 
all-pairs shortest-paths problem, we want to compute the distance between each pair of vertices.

The worst-case complexity of known algorithms for the single-source shortest-paths problem on a directed graph with vertex set $[n]=\{1, \ldots, n\}$ and $m$ edges depends heavily on whether or not edge lengths are allowed to be negative. In fact, if all edge lengths are non-negative, then Dijkstra's algorithm solves the single-source shortest-paths problem in near-linear time $O(m+n \log n)[8,11]$ and the algorithms of McGeoch [20] and Karger, Koller, and Phillips [18] solve the all-pairs shortest-paths problem in time $O\left(n|H|+n^{2} \log n\right)$, where $H$ is the set of edges that are a shortest path between their endpoints. In the general case, the Bellman-Ford algorithm $[2,10]$ solves the single-source shortest-paths problem in time $O(\nu m)$, where $\nu$ is the maximal number of edges on a shortest path. This is $O(n m)$ in the worst case. The solution of one single-source shortest-paths problem allows to transform a problem with arbitrary real edge lengths into an equivalent problem with non-negative edge lengths $[9,17]$. This gives a running time of $O\left(n m+n^{2} \log n\right)$ for the all-pairs shortest-paths problem in the general case. Somewhat better running times are known if the edge lengths are assumed to be integers from some fixed range; see $[1,13,5]$.

Worst-case analysis, however, sometimes fails to bring out the advantages of algorithms that perform well in practice; average-case analysis has turned out to be more appropriate for these purposes. In average-case analysis, we study the expected ruming time of shortest-paths algorithms where instances of shortest-paths problems are generated according to a probability distribution on the set of complete directed graphs with edge lengths. Two kinds of probability distributions have been considered in the literature. In the uniform model, the edge lengths are independent, identically distributed random variables. The endpoint-independent model is more general. A random instance generated according to the endpoint-independent model has the property that if the edges leaving a vertex are sorted according to their lengths, then the associated endpoints occur in random order. This even includes the case that the edge lengths themselves are arbitrarily fixed, and only the assignment of the edge lengths to the edges leaving a vertex is random.

The average running time of shortest-paths algorithms has mainly been studied for the case of non-negative edge lengths. In the endpoint-independent model, the following results on the average-case complexity of the singlesource shortest-paths problem (on instances with $n$ vertices) are known. Noshita [23] analyzed the average-case complexity of Dijkstra's algorithm; the time bound, however, does not improve over the worst-case complexity 
of the algorithm. Spira [24] dealt first with the average-case complexity of the all-pairs shortest-paths problem. He proved an expected time bound of $O\left(n^{2}(\log n)^{2}\right)$, which was later improved by Bloniarz [3] and Frieze and Grimmett [12]. Moffat and Takaoka [22] described an algorithm with expected rumning time $O\left(n^{2} \log n\right)$. Recently, Mehlhorn and Priebe [21] showed that the algorithm of Moffat and Takaoka is reliable, that is, it runs in time $O\left(n^{2} \log n\right)$ with high probability and not just in expectation. In the uniform model, Frieze and Grimmett [12] derived precise results on the distribution of edge lengths and distances. It is a consequence of their results that the expected running time of the algorithms of McGeoch and Karger et al. is $O\left(n^{2} \log n\right)$ in the uniform model.

Concerning the average-case complexity in the case of arbitrary real edge lengths, it is a major problem to define a probability distribution that allows negative edge lengths but does not trivialize the problem. For an instance of the shortest-paths problem on a directed graph $D$, let $D_{\geq 0}$ and $D_{<0}$ be the subgraphs formed by the edges of non-negative and negative length, respectively. If $D_{<0}$ contains a cycle and $D_{\geq 0}$ consists of a single strongly connected component, then all distances are $-\infty$. Topological sorting allows to decide in linear time whether $D_{<0}$ contains a cycle, and depth-first search allows to decide in linear time whether $D_{\geq 0}$ consists of a single strongly connected component. We conclude that shortest-paths problems become trivial if the probability distribution is such that, with high probability, $D_{<0}$ contains a cycle and $D_{\geq 0}$ consists of a single strongly connected component. This will, for example, be the case if edge lengths have a non-zero probability of being negative and instances are generated according to the uniform model.

To the best of our knowledge, a probability distribution for graphs with arbitrary real edge lengths (in order to generate instances of shortest-paths problems) was proposed in the recent paper by Kolliopoulos and Stein [19] for the first time. They studied the endpoint-independent model and gave an algorithm for the single-source shortest-paths problem with expected running time $O\left(n^{2} \log n\right)$. In general, it is not clear how likely a negative cycle is in the endpoint-independent model. (Note, however, that if each vertex has at least one outgoing edge of negative length, then the directed graph must contain a negative cycle.)

We study the average-case complexity of shortest-paths problems in the vertex-potential model. This model was used previously by Cherkassky, Goldberg, and Radzik [4] in an experimental evaluation of shortest-paths algorithms. In this model, there is a potential $\pi_{i}$ for each vertex $i \in[n]$ and a random variable $r_{i, j}$ for each edge $(i, j), i, j \in[n]$. (We will set $r_{i, i} \equiv 0$, 
for $i \in[n]$.$) The edge lengths are defined by$

$$
c_{i, j}=r_{i, j}-\pi_{i}+\pi_{j}, \text { for all edges }(i, j) .
$$

Of course, only the $c_{i, j}$ 's are revealed to our algorithms and the $r_{i, j}$ 's and $\pi_{i}$ 's are hidden parameters of the model. The variables $r_{i, j}, i, j \in[n], i \neq j$, are assumed to be independent, identically distributed random variables with values in $[0,1]$. (Some additional assumptions on the common distribution function $F$ of the $r_{i, j}$ 's are needed to allow the application of the results of Frieze and Grimmett [12]. This will be made more precise in Sect. 3.) The assumption $r_{i, j} \geq 0, i, j \in[n]$, guarantees that instances generated according to the vertex-potential model contain no negative cycle; see Propositions 1 and 2 . The vertex potentials $\pi_{i}, i \in[n]$, may be arbitrarily chosen, provided that they are contained in a real interval of size $O\left(n /(\log n)^{2}\right)$. (This is a considerable generalization of the model that we studied in [6], where the $\pi_{i}$ 's were assumed to be random variables with values in $[-1,1]$.)

We show that the single-source shortest-paths problem can be solved in $O\left(n^{2}\right)$ expected time and that the all-pairs shortest-paths problem can be solved in $O\left(n^{2} \log n\right)$ expected time. In both cases our algorithms are reliable, that is, finish their computations within the respective time bounds with high probability. Our algorithm for the single-source shortest-paths problem first computes vertex potentials $\widehat{\pi}_{i}, i \in[n]$, with the property that for any $i, j \in[n]$, the difference $\widehat{\pi}_{i}-\widehat{\pi}_{j}$ is close to $\pi_{i}-\pi_{j}$. The $\widehat{\pi}_{i}$ 's are then used to define reduced edge lengths $\widehat{c}$ by $\widehat{c}_{i, j}:=c_{i, j}+\widehat{\pi}_{i}-\widehat{\pi}_{j}$. The reduced edge lengths will not be non-negative, they do however allow the extraction of a small number of relevant edges from the complete graph that, with high probability, give the same shortest-path distances. We extract $O\left(n^{2} /(\log n)^{2}\right)$ edges and run the Bellman-Ford algorithm on this edge set. We also argue that each shortest path consists of at most $O\left((\log n)^{2}\right)$ edges with high probability. Taking the two facts together, we get a solution to one single-source shortest-paths problem in expected time $O\left(n^{2}\right)$. We use this solution to define non-negative edge lengths and then use the algorithm of McGeoch or Karger, Koller, and Phillips to solve the all-pairs shortest-paths problem in $O\left(n^{2} \log n\right)$ expected time.

The vertex-potential model and the endpoint-independent model are incomparable as the endpoint-independent model cannot exclude negative cycles and the vertex-potential model excludes negative cycles. Moreover, in the vertex-potential model, there is a strong correlation between the endpoint of an edge and the length of the edge. Assume that the distribution function $F$ is such that all $r_{i, j}$ 's are very close to zero. This implies that for 
all vertices $i$, it is probable that the shortest edge leaving $i$ will go to the vertex with minimum potential.

The paper is organized as follows. In Sect. 2, we recall basic facts about shortest paths and reduced edge lengths. We provide auxiliary results needed for the analysis of our algorithms in Sect. 3. The algorithms and their analysis are presented in Sect. 4.

\section{Shortest paths and reduced edge lengths}

Let $D_{n}$ be the complete loop-less directed graph on the set $[n]=\{1, \ldots, n\}$ of vertices. Assume that edge lengths are given by a function $c$ from the set of edges to the reals; the length of the directed edge $(i, j)$ will be denoted by $c_{i, j}$, for $i, j \in[n]$. (For convenience, let $c_{i, i} \equiv 0$, for $i \in[n]$.) We will write $\left(D_{n}, c\right)$ for $D_{n}$ with edge lengths $c$.

For a directed path $P$ in $D_{n}$, let $c(P)$ be the length of $P$ with respect to $c$, that is, $c(P)$ is defined as $\sum_{(i, j) \in P} c_{i, j}$. For any pair $i, j$ of vertices, let $\delta_{i, j}(c)$ be the infimum of the lengths of all paths from $i$ to $j$. The quantity $\delta_{i, j}(c)$ will be referred to as the distance of $j$ from $i$ (with respect to $c$ ).

We consider the following two shortest-path problems. For a given source vertex $s \in[n]$, the single-source shortest-paths problem asks for the distances of all vertices $i \in[n]$ from $s$. If $s$ is fixed and no confusion is possible, we will denote these distances by $\delta_{i}(c)$ for $i \in[n]$. In the all-pairs shortest-paths problem, we want to compute the distance between each pair of vertices. The maximum of all these distances is called the diameter of $D_{n}$ with respect to $c$ and will be denoted by $\Delta(c)$.

As mentioned in the introduction, we want to generate instances of shortest-paths problems without negative cycles. The following proposition gives a characterization of edge lengths $c$ for which $\left(D_{n}, c\right)$ does not contain a negative cycle.

Proposition 1 The absence of negative cycles in $\left(D_{n}, c\right)$ is equivalent to the existence of vertex potentials $\pi_{i} \in \mathbb{R}, i \in[n]$, so that the reduced edge lengths $r$ (of $c$ with respect to the $\pi_{i}$ 's) are non-negative, that is,

$$
r_{i, j}:=c_{i, j}+\pi_{i}-\pi_{j} \geq 0, \text { for all edges }(i, j) .
$$

Proposition 1 relies on the following optimality conditions for a solution to the single-source shortest-paths problem (with source $s$ ).

For every vertex $i \in[n]$, let $d_{i}(c)$ denote the length (with respect to $c$ ) of some directed path from $s$ to $i$, with $d_{s}(c)=0$. The 
quantities $d_{i}(c)$ are equal to the distances $\delta_{i}(c)$ if and only if they satisfy

$$
d_{i}(c)+c_{i, j} \geq d_{j}(c), \text { for all edges }(i, j) .
$$

It is well-known that shortest-paths problems are invariant under reduction of edge lengths with respect to vertex potentials. We summarize this knowledge in the following proposition.

Proposition 2 Suppose that we associate a vertex potential $\widehat{\pi}_{i} \in \mathbb{R}$ with each vertex $i \in[n]$ and that we define reduced edge lengths $\widehat{c}$ (of $c$ with respect to the $\widehat{\pi}_{i}^{\prime}$ 's) by $\widehat{c}_{i, j}:=c_{i, j}+\widehat{\pi}_{i}-\widehat{\pi}_{j}$ for any edge $(i, j)$. Then, for any directed path $P$ from vertex $k$ to vertex $\ell$,

$$
\widehat{c}(P)=\sum_{(i, j) \in P}\left(c_{i, j}+\widehat{\pi}_{i}-\widehat{\pi}_{j}\right)=c(P)+\widehat{\pi}_{k}-\widehat{\pi}_{\ell} .
$$

Therefore, distances with respect to $c$ and $\widehat{c}$ relate to each other through $\delta_{k, \ell}(\widehat{c})=\delta_{k, \ell}(c)+\widehat{\pi}_{k}-\widehat{\pi}_{\ell}$, for all $k, \ell \in[n]$. It also follows from (2) that, for any directed cycle $C, \sum_{(i, j) \in C} \widehat{c}_{i, j}=\sum_{(i, j) \in C} c_{i, j}$; in particular, $\left(D_{n}, \widehat{c}\right)$ contains a negative cycle if and only if there is a negative cycle in $\left(D_{n}, c\right)$.

\section{Properties of the vertex-potential model}

In the vertex-potential model that we consider in this paper, edge lengths $c_{i, j}$ are generated according to

$$
c_{i, j}=r_{i, j}-\pi_{i}+\pi_{j}, \text { for all edges }(i, j),
$$

with random variables $r_{i, j} \geq 0$ and (not necessarily random) vertex potentials $\pi_{i}, i, j \in[n]$. (We will set $r_{i, i} \equiv 0$, for $i \in[n]$.) Our precise assumptions for these variables are as follows:

(A1) The variables $r_{i, j}, i, j \in[n], i \neq j$, are independent, identically distributed random variables with values in $[0,1]$ and mean $\rho$. We will denote their common distribution function by $F$ and we assume that $F(0)=0$ and that $F^{\prime}(0)$ exists and is strictly positive. (This implies $\rho>0$.)

(A2) The vertex potentials $\pi_{i}, i \in[n]$, are arbitrary real numbers from $[-1,1]$. 
The interval $[-1,1]$ in (A2) is chosen for ease of presentation only. It will be apparent from our proofs that only the size of the interval, from which the vertex potentials are chosen, is important, and that the size may actually be as large as $O\left(n /(\log n)^{2}\right)$; see Remark 2 in Sect. 4.1. Note that even (A2) subsumes the assumption on the $\pi_{i}$ 's in [6], where they were assumed to be independent, identically distributed random variables with values in $[-1,1]$.

The independence assumptions in (A1) are the most important (and restrictive) ones. We will not need to know the value of $\rho$; if needed, we could get a good estimate for it from the data, see the remark after Lemma 4. This lemma will be the only place where we use that the random variables are bounded, and the assumption of boundedness is there more for convenience than necessity. It could be replaced by bounds on the tails of the distributions. By definition of $F^{\prime}(0)$ and since $F(0)=0$,

$$
F(\varepsilon)=\operatorname{Pr}\left(r_{i, j} \leq \varepsilon\right)=F^{\prime}(0) \cdot \varepsilon+o(\varepsilon), \quad \text { as } \varepsilon \rightarrow 0,
$$

that is, the assumption $F^{\prime}(0)>0$ implies that the distribution of the $r_{i, j}$ 's can be approximated by uniform distributions in a neighborhood of 0 . This allows to reduce the proof of Lemma 1 to the case of the uniform distribution on $[0,1]$.

For a problem of size $n$, we will say that an event occurs with high probability if it occurs with probability $\geq 1-O\left(n^{-\gamma}\right)$ for an arbitrary but fixed constant $\gamma \geq 1$. To ensure a probability of failure $O\left(n^{-\gamma}\right)$, in most of our statements, we have to choose sufficiently large constants, depending on the actual value of $\gamma$. This is sometimes made explicit by a subscript $\gamma$.

\subsection{The non-negative case $(\pi \equiv 0)$}

If the $r_{i, j}$ 's are distributed as in (A1) and the $\pi_{i}$ 's are identically 0 , then this gives rise to an instance $\left(D_{n}, r\right)$ with non-negative edge lengths, and the analysis of shortest-paths algorithms by Frieze and Grimmett in [12] can be applied. We briefly review how they argue to obtain bounds on the distances in $\left(D_{n}, r\right)$. For every vertex $i$, they construct a spanning tree $T_{i}$ rooted at $i$. With high probability, the length of the path in $T_{i}$ from the root to any other vertex is $O((\log n) / n)[12,(4.6)$ and (4.14)]. This implies that the diameter $\Delta(r)$ is $O((\log n) / n)$ with high probability. (Davis and Prieditis [7] showed that for exponentially distributed $r$, the expected length of a shortest path is of exactly this order of magnitude. This is also true for $r$ uniformly distributed; see $[7,20]$.)

Furthermore, if $(i, j)$ is the $p$-th shortest edge in the adjacency list of $i$ where $p>B_{\gamma} \log n$ (for a sufficiently large constant $B_{\gamma}$ ), then $r_{i, j}>$ 
$\Delta(r)$ with high probability [12, Lemma 4.3]. This means that, with high probability, edge $(i, j)$ is irrelevant, that is, is not contained in any shortest path in $\left(D_{n}, r\right)$. We can therefore restrict ourselves to examining the sparse graph with only the $O(\log n)$ shortest edges from each adjacency list. We will show in Lemma 5 how to adjust this idea of sparsifying the graph if edge lengths are distributed according to the vertex-potential model.

Lemma 1 ([12]) Suppose that edge lengths in $\left(D_{n}, r\right)$ are distributed as specified in (A1). Then, with high probability, $\Delta(r)=O((\log n) / n)$, and the set $A:=\left\{(i, j) ; r_{i, j} \leq \Delta(r)\right\}$ of relevant edges is of cardinality $O(n \log n)$.

(For the special case of uniformly distributed $r$, similar results were proved by Hassin and Zemel in [15].) By a fairly involved argument, Frieze and Grimmett also proved that each of the trees $T_{i}, i \in[n]$, is of depth $O(\log n)$, that is, that the short (in length, though not necessarily shortest-length) paths in $T_{i}$ consist of $O(\log n)$ edges with high probability [12, Theorem 5.2]. Shortest paths may have more (but shorter) edges, but we now prove that, with high probability, shortest paths also consist of few edges only.

Lemma 2 With high probability, shortest paths in $\left(D_{n}, r\right)$ consist of $O\left((\log n)^{2}\right)$ edges.

Proof. This will follow from the fact that if at least $|P| / k$ of the edges of a shortest path $P$ in $\left(D_{n}, r\right)$ have length at least $\alpha$, then $|P| \leq k \cdot \Delta(r) / \alpha$, where $|P|$ denotes the number of edges in $P$.

By (3), we can fix $\alpha=\Theta(1 / n)$ so that $p_{\alpha}=\operatorname{Pr}\left(r_{i, j} \leq \alpha\right) \leq 1 /(2 n)$ for sufficiently large $n$; an edge $(i, j)$ with $r_{i, j} \leq \alpha$ will be called tiny. For any $k \geq 1$, the probability that a (directed) path with $k$ edges consists only of tiny edges is $\leq p_{\alpha}{ }^{k}$. Hence, with probability $\geq 1-2\left(\begin{array}{c}n \\ k+1\end{array}\right) p_{\alpha}{ }^{k} \geq 1-n / 2^{k}$, no path in $\left(D_{n}, r\right)$ with $\geq k$ edges consists only of tiny edges, which implies that any path $P$ in $\left(D_{n}, r\right)$ has at least $\lfloor|P| / k\rfloor$ edges of lengths $>\alpha$. If we set $k=K_{\gamma} \log n$ with $K_{\gamma}$ chosen large enough, then, together with the bound on $\Delta(r)$ from Lemma 1 , we conclude that $|P|=O\left((\log n)^{2}\right)$ for any shortest path $P$ in $\left(D_{n}, r\right)$ with high probability.

Since shortest paths are invariant under reduction of edge lengths with respect to vertex potentials (see Section 2), the following is an immediate consequence of Lemma 2.

Corollary 1 Let edge lengths $r$ be distributed as specified in (A1), and let edge lengths $c$ be obtained by reducing the edge lengths $r$ with respect to some 
vertex potentials. Shortest paths in $\left(D_{n}, c\right)$ consist then of $O\left((\log n)^{2}\right)$ edges with high probability.

\subsection{The general case: Approximating the vertex-potential differences}

We will use the following form of the well-known Chernoff-Hoeffding bound on the tail of the distribution of a sum of independent random variables; see $[16,14]$ for a proof.

Lemma 3 Let $X$ be the sum of independent, identically distributed random variables $X_{1}, \ldots, X_{m}$ with values in $[0,1]$; let $\xi:=E\left[X_{1}\right]$. Then, for any $\varepsilon$, $0<\varepsilon<1$,

$$
\operatorname{Pr}(|X / m-\xi|>\varepsilon \xi) \leq 2 \cdot e^{-\varepsilon^{2} m \xi / 3}
$$

We now show how to compute vertex potentials $\widehat{\pi}_{i}, i \in[n]$, so that, for any $k, \ell \in[n]$, with high probability, the difference $\widehat{\pi}_{k}-\widehat{\pi}_{\ell}$ is a good approximation of the actual vertex-potential difference $\pi_{k}-\pi_{\ell}$.

Lemma 4 For any $i \in[n]$, define

$$
\widehat{\pi}_{i}:=-\frac{1}{n-1} \cdot \sum_{j=1}^{n} c_{i, j} .
$$

Then, for any $k, \ell \in[n]$, the term $\left|\left(\widehat{\pi}_{k}-\widehat{\pi}_{\ell}\right)-\left(\pi_{k}-\pi_{\ell}\right)\right|$ is of order $O(\sqrt{(\log n) / n})$ with high probability.

Proof. For any $k, j \in[n]$, we have $-c_{k, j}=\pi_{k}-r_{k, j}-\pi_{j}$ by definition; thus,

$$
-\frac{1}{n-1} \cdot \sum_{j=1}^{n} c_{k, j}=\frac{n}{n-1} \cdot \pi_{k}-\frac{1}{n-1} \cdot \sum_{j=1}^{n} r_{k, j}-\frac{1}{n-1} \cdot \sum_{j=1}^{n} \pi_{j}
$$

from which we conclude that

$$
\widehat{\pi}_{k}-\pi_{k}=\frac{1}{n-1} \cdot \pi_{k}-\frac{1}{n-1} \cdot \sum_{j=1}^{n} r_{k, j}-\frac{1}{n-1} \cdot \sum_{j=1}^{n} \pi_{j} .
$$

Note that the rightmost term, $\frac{1}{n-1} \cdot \sum_{j=1}^{n} \pi_{j}$, is independent of $k$. Hence, for any $k, \ell \in[n]$,

$$
\left|\left(\widehat{\pi}_{k}-\pi_{k}\right)-\left(\widehat{\pi}_{\ell}-\pi_{\ell}\right)\right| \leq \frac{1}{n-1} \cdot\left|\pi_{k}-\pi_{\ell}\right|+2 \max _{i}\left|\frac{1}{n-1} \cdot \sum_{j=1}^{n} r_{i, j}-\rho\right|,
$$


where $\rho=\frac{1}{n-1} \cdot E\left[\sum_{j} r_{i, j}\right]$. By assumption (A1), $\sum_{j} r_{i, j}$ is the sum of $n-1$ independent, identically distributed random variables with values in $[0,1]$, and hence, the Chernoff-Hoeffding bound (4) implies that, with high probability,

$$
\left|\frac{1}{n-1} \cdot \sum_{j} r_{i, j}-\rho\right|=O(\sqrt{(\rho \cdot \log n) / n})=O(\sqrt{(\log n) / n}),
$$

for any $i \in[n]$. Thus $2 \max _{i}\left|\frac{1}{n-1} \cdot \sum_{j} r_{i, j}-\rho\right|=O(\sqrt{(\log n) / n})$ with high probability. The other term on the right-hand side of (5) is of order $O(1 / n)$ by assumption (A2). This proves that $\left|\left(\widehat{\pi}_{k}-\widehat{\pi}_{\ell}\right)-\left(\pi_{k}-\pi_{\ell}\right)\right|$ is $O(\sqrt{(\log n) / n})$ with high probability.

Remark 1 In [6], we defined approximate vertex potentials $\widehat{\pi}_{i}=\widehat{\rho}-\frac{1}{n-1}$. $\sum_{j} c_{i, j}$, for $i \in[n]$, where $\widehat{\rho}=\frac{1}{n(n-1)} \cdot \sum_{i, j} c_{i, j}=\frac{1}{n(n-1)} \cdot \sum_{i, j} r_{i, j}$. (The "observed mean" $\widehat{\rho}$ is a good approximation of $\rho$.) Using the stronger assumptions of $[6]$ on the $\pi_{i}$ 's, one can prove that $\left|\widehat{\pi}_{i}-\pi_{i}\right|=O(\sqrt{(\log n) / n})$ with high probability. It turns out, however, that the approximation of single vertex potentials is not needed in the analysis of our algorithms; see Sect. 4.1.

\section{The algorithms}

\subsection{Solving the single-source shortest-paths problem}

We are now ready to explain in detail how we solve an instance $\left(D_{n}, c\right)$ of the single-source shortest-paths problem if edge lengths in $\left(D_{n}, c\right)$ are generated according to the vertex-potential model of Sect. 3. Our algorithm proceeds in three phases, a preprocessing phase, a computation phase, and a postprocessing phase, in which the correctness of the solution from the second phase is checked. Let the source vertex for the single-source shortestpaths problem under consideration be denoted by $s$.

Preprocessing. The algorithm computes, for every vertex $i \in[n]$, a vertex potential $\widehat{\pi}_{i}$ as in Lemma 4 and transforms the edge lengths $c_{i, j}$ to

$$
\widehat{c}_{i, j}:=c_{i, j}+\widehat{\pi}_{i}-\widehat{\pi}_{j}=r_{i, j}+\left(\widehat{\pi}_{i}-\pi_{i}\right)-\left(\widehat{\pi}_{j}-\pi_{j}\right) \text {, for all edges }(i, j) \text {. }
$$

The shortest-paths problems $\left(D_{n}, c\right)$ and $\left(D_{n}, \widehat{c}\right)$ are equivalent. However, $\left(D_{n}, \widehat{c}\right)$ is more efficiently solvable, since edge lengths $\widehat{c}$ allow a substantial sparsification of the underlying graph. The following lemma gives a bound on the length of edges that are irrelevant with respect to $\widehat{c}$. 
Lemma 5 If $\widehat{c}_{i, j}>\Delta(r)+\max _{k, \ell}\left|\left(\widehat{\pi}_{k}-\pi_{k}\right)-\left(\widehat{\pi}_{\ell}-\pi_{\ell}\right)\right|$, then edge $(i, j)$ is not contained in any shortest path.

Proof. If $(i, j)$ is contained in a shortest path, then $\widehat{c}_{i, j}=\delta_{i, j}(\widehat{c}) \leq \Delta(\widehat{c})$. It follows from (6) and Proposition 2 that for any $k, \ell \in[n], \delta_{k, \ell}(\widehat{c})=\delta_{k, \ell}(r)+$ $\left(\widehat{\pi}_{k}-\pi_{k}\right)-\left(\widehat{\pi}_{\ell}-\pi_{\ell}\right)$. This implies $\Delta(\widehat{c}) \leq \Delta(r)+\max _{k, \ell}\left|\left(\widehat{\pi}_{k}-\pi_{k}\right)-\left(\widehat{\pi}_{\ell}-\pi_{\ell}\right)\right|$.

For $\gamma \geq 1$ arbitrary but fixed, we know from Lemma 1 and from Lemma 4 that there exist constants $C_{\gamma}$ and $M_{\gamma}$ so that

$\Delta(r) \leq C_{\gamma}(\log n) / n \quad$ and $\quad \max _{k, \ell}\left|\left(\widehat{\pi}_{k}-\pi_{k}\right)-\left(\widehat{\pi}_{\ell}-\pi_{\ell}\right)\right| \leq M_{\gamma} \sqrt{(\log n) / n}$

with probability $\geq 1-O\left(n^{-\gamma}\right)$. For the time being, we will assume that (7) holds. For an arbitrary (but fixed) constant $L_{\gamma}>M_{\gamma}$ (and sufficiently large $n$ ), all relevant edges (that is, edges that will possibly be contained in a shortest path) are then contained in

$$
\widehat{A}:=\left\{(i, j) ; \widehat{c}_{i, j} \leq L_{\gamma} \sqrt{(\log n) / n}\right\} .
$$

(A more careful proof of Lemma 4 reveals that we could set $L_{\gamma}=5 \sqrt{\gamma}$. This value is not optimal but nevertheless indicates that, for given $\gamma$, some explicit constant $L_{\gamma}$ is easily derivable.) The vertex potentials $\widehat{\pi}_{i}, i \in[n]$, the reduced edge lengths $\widehat{c}$, and the set $\widehat{A}$ can be computed in $O\left(n^{2}\right)$ time. Let $\widehat{D}$ denote the graph $([n], \widehat{A})$.

Computation. We now solve a single-source shortest-paths problem with source $s$ on the sparsified graph $(\widehat{D}, \widehat{c})$ by running the Bellman-Ford algorithm $[2,10]$. This algorithm maintains tentative distances $d_{i}$ for every vertex $i \in[n]$. The $d_{i}$ 's are initially set to $\infty$ (except for $\left.d_{s}=0\right)$, and $d_{i}$ always represents the length of some path in $(\widehat{D}, \widehat{c})$ from $s$ to $i$. The Bellman-Ford algorithm proceeds in passes over the edge set $\widehat{A}$, maintaining the following invariant. After the $k$-th pass, the Bellman-Ford algorithm has correctly computed the distances of all vertices to which there is a shortest path from $s$ consisting of at most $k$ edges. The algorithm actually checks the optimality conditions (1) for all edges (in $\widehat{A}$ ) in each pass, and it will therefore terminate (with all distances in $(\widehat{D}, \widehat{c})$ computed correctly) after the $\nu$-th pass, where $\nu$ is the maximum number of edges in a shortest path in $(\widehat{D}, \widehat{c})$. The running time of the Bellman-Ford algorithm is therefore $O(\nu \cdot|\widehat{A}|)$, which is $O\left(n^{3}\right)$ in the worst case but $o\left(n^{2}\right)$ with high probability, as we now argue. 
By (6) and (7),

$$
\widehat{A} \subseteq\left\{(i, j) ; r_{i, j} \leq\left(L_{\gamma}+M_{\gamma}\right) \sqrt{(\log n) / n}\right\},
$$

and it follows from (3) that edge $(i, j)$ is an element of the set on the righthand side with probability $\widehat{p}=\Theta(\sqrt{(\log n) / n})$ for sufficiently large $n$, independently of all the other edges. The random variable $|\widehat{A}|$, the cardinality of $\widehat{A}$, is therefore stochastically dominated by a random variable that is binomially distributed with parameters $n(n-1)$ and $\widehat{p}$. We apply the tail estimates of Lemma 3 to deduce that $|\widehat{A}|=O(n(n-1) \sqrt{(\log n) / n})=O\left(n^{3 / 2} \sqrt{\log n}\right)$ with high probability. By (6) and Corollary $1, \nu=O\left((\log n)^{2}\right)$ with high probability. Hence, with high probability, it takes $O\left(n^{3 / 2}(\log n)^{5 / 2}\right)=o\left(n^{2}\right)$ time to run the Bellman-Ford algorithm on the sparsified graph $(\widehat{D}, \widehat{c})$.

Postprocessing. The second phase will have failed to compute all distances correctly only if (7) does not hold, which happens only with probability $O\left(n^{-\gamma}\right)$. The optimality conditions (1) (checked for all edges) are an $O\left(n^{2}\right)$-time certificate for the correctness of the solution. Since the worstcase rumning time of the Bellman-Ford algorithm on $\left(D_{n}, \widehat{c}\right)$ is $O\left(n^{3}\right)$, we can easily afford to run the Bellman-Ford algorithm on $\left(D_{n}, \widehat{c}\right)$ in case of failure, without affecting the bounds on the ruming time. Finally, it takes $O\left(n^{2}\right)$ time to compute the distances $\delta_{i}(c)=\delta_{i}(\widehat{c})-\widehat{\pi}_{s}+\widehat{\pi}_{i}, i \in[n]$.

The discussion above is summarized in the following theorem.

Theorem 1 Assume that edge lengths in $\left(D_{n}, c\right)$ are generated according to the vertex-potential model of Sect. 3. The single-source shortest-path problem can then be solved in time $O\left(n^{2}\right)$ with high probability.

Remark 2 Theorem 1 will still hold if the Bellman-Ford algorithm takes time $O\left(n^{2}\right)$ on $(\widehat{D}, \widehat{c})$ (with high probability), since pre- and postprocessing of the algorithm take time $O\left(n^{2}\right)$ anyway. This means that because of Corollary 1 , we only have to ensure that, with high probability, the set of relevant edges (with respect to $\widehat{c}$ ) is of cardinality at most $O\left(n^{2} /(\log n)^{2}\right)$. Perhaps surprisingly, these observations allow us to relax (A2) to the much weaker assumption

$$
\max _{i, j}\left|\pi_{i}-\pi_{j}\right|=O\left(n /(\log n)^{2}\right)
$$

Note that the term $\left|\pi_{k}-\pi_{\ell}\right| /(n-1)=O\left(1 /(\log n)^{2}\right)$ will then dominate the right-hand side of (5) so that $\max _{k, l}\left|\left(\widehat{\pi}_{k}-\pi_{k}\right)-\left(\widehat{\pi}_{\ell}-\pi_{\ell}\right)\right|=O\left(1 /(\log n)^{2}\right)$ with high probability. Using arguments as in the proofs of Lemma 5 and 
Theorem 1, one can then show that the algorithm of Bellman and Ford will compute correct distances (with respect to $\widehat{c}$ ) with high probability if it is restricted to the edges in $\left\{(i, j) ; \widehat{c}_{i, j} \leq R /(\log n)^{2}\right\}$, for a suitable constant $R$. With high probability, this is indeed a set of cardinality $O\left(n^{2} /(\log n)^{2}\right)$.

\subsection{Solving the all-pairs shortest-paths problem}

Theorem 2 Assume that the edge lengths in $\left(D_{n}, c\right)$ are generated according to the vertex-potential model of Sect. 3. The all-pairs shortest-path problem can then be solved in $O\left(n^{2} \log n\right)$ with high probability.

Proof. We first compute distances $\delta_{i}(c), i \in[n]$, with respect to source vertex 1 by solving a single-source shortest-paths problem as in the proof of Theorem 1 . This takes time $O\left(n^{2}\right)$ with high probability. Let $\bar{c}$ be the reduced edge lengths of $c$ with respect to the vertex potentials $\delta_{i}(c), i \in[n]$, that is, for all edges $(i, j)$,

$$
\begin{aligned}
\bar{c}_{i, j} & =c_{i, j}+\delta_{i}(c)-\delta_{j}(c) \\
& =r_{i, j}+\left(\delta_{i}(c)-\pi_{i}\right)-\left(\delta_{j}(c)-\pi_{j}\right) .
\end{aligned}
$$

It follows from the first equality and the optimality conditions (1), that $\bar{c}_{i, j} \geq 0$ for all edges $(i, j)$. The reduced edge lengths $\bar{c}$ can be computed in $O\left(n^{2}\right)$ time, and the same time bound will allow to transform distances $\delta_{i, j}(\bar{c})$ into distances $\delta_{i, j}(c)$, for all $i, j \in[n]$.

To compute the $\delta_{i, j}(\bar{c})$ 's, we run one of the algorithms of Karger, Koller, and Phillips [18] or McGeoch [20] on $\left(D_{n}, \bar{c}\right)$ that efficiently solve the all-pairs shortest-paths problem with non-negative edge lengths. Both algorithms run in time $O\left(n^{2} \log n+n|H|\right)$ where $H=H(\bar{c})$ is the set of edges that are the shortest path (with respect to $\bar{c}$ ) between their endpoints. We apply the arguments of Sect. 2 again. Shortest paths are invariant under reduction of the edge lengths with respect to vertex potentials, and it follows from (8) that the edges in $H$ are also the shortest path between their endpoints with respect to edge lengths $r . H$ is therefore contained in the set $A:=$ $\left\{(i, j) ; r_{i, j} \leq \Delta(r)\right\}$, and it follows by Lemma 1 that $|H|=O(n \log n)$ with high probability. This yields a rumning time of $O\left(n^{2} \log n\right)$ with high probability for the algorithms by McGeoch and Karger, Koller, and Phillips. Since $|H|=O\left(n^{2}\right)$ in the worst case, our algorithm has a running time of $O\left(n^{3}\right)$ with probability $O\left(n^{-\gamma}\right), \gamma \geq 1$, which still gives an expected running time of $O\left(n^{2} \log n\right)$. 


\section{References}

[1] R. K. Ahuja, K. Mehlhorn, J. B. Orlin, and R. E. Tarjan, Faster algorithms for the shortest path problem, J. Assoc. Comput. Mach. 37 (1990) 213-223

[2] R. Bellman, On a routing problem, Quart. Appl. Math. 16 (1958) 87-90

[3] P. A. Bloniarz, A shortest-path algorithm with expected time $O\left(n^{2} \log n \log ^{*} n\right)$, SIAM J. Comput. 12 (1983) 588-600

[4] B. V. Cherkassky, A. V. Goldberg, and T. Radzik, Shortest paths algorithms: Theory and experimental evaluation, Math. Programming 73 (1996) 129-174

[5] B. V. Cherkassky, A. V. Goldberg, and C. Silverstein, Buckets, heaps, lists, and monotone priority queues, Proc. 8th Annual ACM-SIAM Symposium on Discrete Algorithms, New Orleans LA, 1997, 83-92

[6] C. Cooper, A. Frieze, K. Mehlhorn, and V. Priebe, Average-case complexity of shortest-paths problems in the vertex-potential model, in: J. Rolim (Ed.), Randomization and Approximation Techniques in Computer Science (Lecture Notes in Comput. Sci.; 1269), Spinger-Verlag, Berlin, 1997, 15-26

[7] R. Davis and A. Prieditis, The expected length of a shortest path, Inform. Process. Lett. 46 (1993) 135-141

[8] E. W. Dijkstra, A note on two problems in connexion with graphs, Numer. Math. 1 (1959) 269-271

[9] J. Edmonds and R. M. Karp, Theoretical improvements in algorithmic efficiency for network flow problems, J. Assoc. Comput. Mach. 19 (1972) 248-264

[10] L. R. Ford, Jr. and D. R. Fulkerson, Flows in Networks, Princeton University Press, Princeton NJ, 1962

[11] M. L. Fredman and R. E. Tarjan, Fibonacci heaps and their uses in improved network optimization algorithms, J. Assoc. Comput. Mach. 34 (1987) 596-615

[12] A. M. Frieze and G. R. Grimmett, The shortest-path problem for graphs with random arc-lengths, Discrete Appl. Math. 10 (1985) 57-77

[13] A. V. Goldberg, Scaling algorithms for the shortest paths problem, SIAM J. Comput. 24 (1995) 494-504

[14] T. Hagerup and C. Rüb, A guided tour of Chernoff bounds, Inform. Process. Lett. $33(1989 / 90)$ 305-308

[15] R. Hassin and E. Zemel, On shortest paths in graphs with random weights, Math. Oper. Res. 10 (1985) 557-564

[16] W. Hoeffding, Probability inequalities for sums of bounded random variables, J. Amer. Statist. Assoc. 58 (1963) 13-30

[17] D. B. Johnson, Efficient algorithms for shortest paths in sparse networks, J. Assoc. Comput. Mach. 24 (1977) 1-13

[18] D. R. Karger, D. Koller, and S. J. Phillips, Finding the hidden path: Time bounds for all-pairs shortest paths, SIAM J. Comput. 22 (1993) 1199-1217

[19] S. G. Kolliopoulos and C. Stein, Finding real-valued single-source shortest paths in $o\left(n^{3}\right)$ expected time, in: W. H. Cunningham, S. T. McCormick, and M. Queyranne (Eds.), Integer Programming and Combinatorial Optimization (Lecture Notes in Comput. Sci.; 1084), Springer-Verlag, Berlin, 1996, 94-104 
[20] C. C. McGeoch, All-pairs shortest paths and the essential subgraph, Algorithmica 13 (1995) 426-441

[21] K. Mehlhorn and V. Priebe, On the all-pairs shortest-path algorithm of Moffat and Takaoka, Random Structures Algorithms 10 (1997) 205-220

[22] A. Moffat and T. Takaoka, An all pairs shortest path algorithm with expected time $O\left(n^{2} \log n\right)$, SIAM J. Comput. 16 (1987) 1023-1031

[23] K. Noshita, A theorem on the expected complexity of Dijkstra's shortest path algorithm, J. Algorithms 6 (1985) 400-408

[24] P. M. Spira, A new algorithm for finding all shortest paths in a graph of positive arcs in average time $O\left(n^{2} \log ^{2} n\right)$, SIAM J. Comput. 2 (1973) 28-32

This research was sponsored in part by National Science Foundation (NSF) grant no. CCR-0122581. 